\title{
The Theory of the Hidden Variable Behind: The Quantization of Duality
}

\author{
Jaime B. Vigo ${ }^{1}$ \\ ${ }^{1}$ The HVB Research Foundation, Department of Research and Development. PO Box 13150, El Paso, Texas \\ 79913, USA
}

Correspondence: Jaime B. Vigo, The HVB Research Foundation, Department of Research and Development. PO Box 13150, El Paso, Texas 79913, USA. E-mail: jvigo@elp.rr.com

Received: February 3, 2012

Accepted: February 20, 2012 Online Published: May 1, 2012

doi:10.5539/apr.v4n2p82

URL: http://dx.doi.org/10.5539/apr.v4n2p82

\begin{abstract}
Modern knowledge in physics is in need of new ideas in order to find answers to questions about the origins of matter, waveparticle duality, time, the Big Bang, black hole singularity, and evolution. As an effort to collaborate with the infusion of new ideas, this investigation was developed based on the philosophy of hidden variables behind (HVB). This HVB theory is originated from re-interpreting the fundamental concepts of energy delocalization from the atomic model of Bohr and the generation of electric charges in particles from the electromagnetic equations of Maxwell. In the theory, it is hypothesized that before the Big Bang, the electric and magnetic energy were initially totally delocalized, that is, spread over an infinite number of energy levels belonging to a tachyon-like parent matrix of matter that moves faster than the speed of light. The matter at this superluminal state is considered to be non-dual, theoretically having its energy acting $100 \%$ as a wave and $0 \%$ as a particle, hereby identified as pure energy. The luminal matter of this universe is considered to be distorted superluminal matter having lower than $100 \%$ delocalized and higher than $0 \%$ localized energy. The HVB theory introduces a new quantum field that attenuates the degree of localization of pure energy to allow particles to have different degrees of duality, so that duality can be scrambled. The model proposes that a Lorentz-like force acts on the fully-delocalized superluminal energy of the pure matter, causing a slowdown of its velocity, becoming localized luminal speed of light. The increase in localized energy leads to greater mass and charge allocation in particles. In the HVB theory, the universe was originated from a vortex formed of distorted energy-waves of the matrix, which created a gradient of attracted waves driven by increased in electromagnetic force, later developing gravity, forming an equilibrated "bubble" of luminal matter in the superluminal matrix, resembling a slower moving eddy on the surface of the rushing waters of a river. The motion of the bubble-universe in the matrix is represented from the wave point of view, in which the universe is entangled energy propagating through the matter of the matrix medium. The matter of the matrix being distorted to form the universe at point $A$ is not the same matter of the matrix being distorted to re-form it at point $B$. This implies that what is really being propagated is the information or instruction of how to distort matrix matter to look like the universe, with the condition of replacing it with a slight increase in entropy. The quantum-controlled attenuation of the degree of localization gives rise to the particle plethora, allows crossing from particles to anti-particles, and explains how isotopes of the same element may decay at different rates. In the HVB theory time is considered another waveparticle. The concept implies that both waveparticles time and light must have non-zero mass. The flow of the waveparticle time is unidirectional, associated with increased disorder. The energy levels of time are organized by locating the occurrence of any event 1 (the cause) at a higher energy level than the immediate occurring event 2 (the effect). Traveling back in time is forbidden due to energy barriers to reach the past and the future. The theory acclaims the establishment of the fourth law of thermodynamics: Energy use is always minimized. This changes the modern view of the parallel universes. Black holes are seen as places of the matrix in which the Big Bang effect is reversed at a lower scale by returning localized energy back to delocalized, in which the singularity is seen as the place at which luminal physics collapse and superluminal physics takes over.
\end{abstract}

Keywords: antiparticles, big bang, black hole, Bohr, charge, dark matter, Dirac, duality, Einstein, electromagnetic, energy, energy delocalization, energy levels, energy localization, entropy, enthalpy, evolution, Heisenberg, hidden variable Isotopes, light, Lorentz, Luminal, Mass, matrixenes, Maxwell, Maxwell violation, momentum, origins, Parallel Universes, particle, Planck, Podolsky, quanta, quantum field, quantum gravity, 
quarks, radioactive decay, Rosen, Singularity, speed of Light, superluminal, tachyon, thermodynamics, time, time duality, time travel, uncertainty, universe, wavefunction, wavefunction collapse, wavelength, waveparticle, waveparticle time, wave.

\section{Introduction}

Great scientists from the past have left us with the most intriguing questions about the origins of the universe (Zinkernagel, 2011). In modern times, we teach in science courses the most outstanding theories such as quantum mechanics, relativity, and electromagnetism. These theories have successfully explained relevant aspects about the nature of light and the four fundamental forces of the universe: the strong force, the weak force, the electromagnetic field, and gravity. Modern science and engineering advancements that drive the course of human technology and way of living are based on the discoveries of these theories (Genovese, 2005). Although science works to improve and facilitate human life, it still has a certain number of fundamental questions that remain unanswered such as the origin of matter, gravity, time, the Big Bang, and dark matter (Carroll, 2012; Worsley, 2012; Pankaj, 2009; Baez, 2006; Brooks, 2005; Rynasiewicz, 2004; Callender, 2004; Markosian, 2002; Jammer, 2002; Dowden, 2001; Ferrarese, 2000). Modern theoreticians and philosophers continue to explore new ideas attempting to develop theories that can be corroborated experimentally (Zinkernagel, 2011). One remarkable aspect about new discoveries is that there has been very little advance in theoretical physics since the time in which past scientists such as Lorentz, Bohr, Einstein, Planck, Heisenberg, Maxwell, Dirac, Boltzmann and many others put forth their revolutionary ideas. Fundamental theories are continuously a subject of academic debates and research, daily bread of scientists and philosophers hungry for knowledge of truth. Some trends in modern physics tend to seek help for new ideas from philosophy (Zinkernagel, 2011; Chimisso, 2008; Freire, 2006; d'Agostino, 2003; Richardson, 2003, Schummer, 2003; Butterfield, 2002; Folse, 1995; Dürr, 1995; Honner, 1982).

Any modern scientist who has been deeply searching for answers to fundamental questions may soon have discovered that many theories have been squeezed to the extremes in attempts to provide clearer understanding to fundamental questions, sometimes encountering areas of contradictory "nonsense". Successful quantum mechanics theories have given understanding into the duality of the nature of light by considering photons as sub-atomic units with properties of both particles and waves. In physics, the rule dictates that nothing is supposed to move faster than the speed of light. Quantum also has extended the classical Newton equations of motion to explain the behavior of atomic and sub-atomic particles in general (Levine, 1991). For example, in the Copenhagen Interpretation, first given by Niels Bohr who is considered one of the fathers of quantum physics, objects have two kinds of observables: some that can be observed simultaneously, and some that cannot (Wolf, 1988). The concept is extended by the Dirac's equation which sustains that all particles of matter move at the speed of light through jagged patters that produce the illusion that matter moves slower than the speed of light (Wolf, 1988).

One outstanding theoretical discovery is the wavefunction, represented by the Greek letter $\Psi$, a mathematical expression that describes the state of a quantum system. Because the wavefunction represents mathematically a wave or a representation of vibration, the nature of the wavefunction implies certain mathematical complexities. One most remarkable fact is that a single wave is harmonic, in which it can be represented as the total sum of waves having frequencies that are whole multiples numbers of a fundamental wave. For example, the harmonics of $y=\sin (x)$ are $y=\sin (2 x), y=\sin (3 x), y=\sin (4 x)$, and so on. This implies that the vibrations of particles that humans observe are really a superposition of an infinite number of harmonic components vibrating simultaneously. Very interestingly, it has been found that even human brainwaves also show harmonic behavior, a fact also observed in musical instruments (Glassman, 1999). Other scientists have proposed that the structure and dynamics of the DNA molecular helix can be treated mathematically as a wave (Yakushevich, 2001). The wavefunction reality becomes more complex because linear combinations of individual harmonics are also accepted vibrations contributing to the overall wavefunction. Furthermore, the wavefunction becomes weirder because it also includes imaginary values proceeding from the fact that the square root of minus one $(\sqrt{ }(-1)$ or $i)$ appears in quantum equations. The model implies that the wavefunction is really the sum of all possible real and imaginary outcomes for the event measured by the wavefunction. The representation of the actual observed outcome of the state of a system is mathematically represented as the square of the wavefunction $\left(\Psi^{2}\right)$, a mathematical manipulation that represents a statistical probability that eliminates the imaginary value $i$ (Levine, 1991). In atoms, $\Psi^{2}$ symbolizes the space-time areas called atomic orbitals in which electrons can be found, mathematically known as $s, p, d, f$, etc. (Levine, 1991).

Modern theories have also led to other several bizarre suggestions, in which two most outstanding are time traveling and parallel universes. In time travel, one is expected to go back or forward in time by moving at or 
faster than the speed of light. In the parallel universes, there are other universes in which versions of human beings go on with their lives based on the consequences of choices that we did not choose in this universe. Both of these ideas were considered for the first time decades ago and yet none of them has received any evidence of existence or further clarification.

The purpose of this paper is to philosophically contribute to the concept of hidden variables underlying the quantum theory (Dürr, 1995; Bohm, 1980; Bohm, 1957) by proposing a further quantization to the concept of duality. The concept of hidden variables was originated by Einstein, Podolsky, and Rosen in the famous EPR paradox who argued that quantum mechanics was incomplete (Einstein, 1935). In my words, the concept professes that behind any unexplainable phenomenon there is a variable that scientists still have to discover that would explain the unexplained. This concept appears to be controversial to a theorem known as the Bell inequality (Bell, 1964). The controversies around both concepts continue to be in the actuality a matter of discussions within the physics and philosophy arena (d'Espagnat, 2006; Freire, 2006; Genovese, 2005; Bartels, 2004; Callender, 2004; Healey, 2003).

\section{The Need for the Establishment of a New Quantum Field}

Further clarification to outstanding questions in physics and philosophy may proceed from reconsidering already-accepted laws to create new ideas or eliminate those that may be scientifically incorrect. For example, consider creating a new quantum field based on the phenomenon of energy delocalization and localization. Chemists, for example, are very well knowledgeable about the aromatic character of the benzene ring $\left(\mathrm{C}_{6} \mathrm{H}_{6}\right)$, a hexagonal flat molecule of carbon atoms alternately double-bonded to each other. In it, there are three pairs of specialized bonding electrons called pi $(\pi)$ that appear simultaneously shared between the atomic orbitals of all six carbon atoms forming the ring (Morrison \& Boyd, 1973). These electrons behave distinctively to those forming the single carbon-carbon bonds called sigma $(\sigma)$. The quantum field theory explains that the $\pi$ electrons are capable of moving above and below the flat hexagon ring in circular motion because they are energetically capable of being shared simultaneously as a consequence of the $s p^{2}$ hybridization of the molecular orbitals of the carbon atoms. This behavior opposes the $\sigma$ electrons, which appear "confined" between the atomic orbitals of only two carbon atoms. In the quantum theory of molecular orbitals, it is said that the $\pi$ electrons are delocalized (Morrison \& Boyd, 1973), whereas the $\sigma$ are localized (Levine, 1991). By delocalization it is meant that the energy of the $\pi$ electrons is spread over a larger number of energy levels and thus allowed to be found in several places at the same time, whereas localization means that the energy is spread over a smaller number of energy levels, and thus allowed to be found in less places at the same time. A closer look into physics demonstrates that the property of delocalization is due to the wave nature of the electrons, a consequence of duality. The concept of delocalization is tied to resonance, a phenomenon well known in chemistry and physics, especially in modern applications such as electronics. The importance of electron delocalization to life includes the fact that the frequency at which $\pi$ electrons move in the porphyrin molecule of chlorophyll is what is responsible for capturing the ultraviolet (uv) light from the sun in order to initiate the biological process of photosynthesis in autotrophs organisms such as plants and algae (McGraw-Hill, 1994).

Let us consider introducing a new quantum field based on the concept of delocalization-localization to determine whether the properties of a system, such as the energy, would be spread over a larger or smaller number of energy levels. I drew this idea from the 1800's physicist named Maxwell who unified the electric and the magnetic fields through a set of four mathematical equations (McGraw-Hill, 1994). In physics, it is said that a moving charge generates a perpendicular magnetic field (Serway, 1986). It is curious, but I share with other scientists the impression that the flow of information in textbooks creates in our brains ideas that flow in one direction, in this case, by explaining that the mass of an electrically-charged particle is responsible for creating the perpendicular magnetic field. However, with a little critical thinking, I reversed the flow of the thought and tried to re-interpret it backwards by rather considering the possibility that the magnetic field creates the charged-particle. I have a citation to Maxwell's work that gives me the hunch that magnetic waves have something that the electric charges do not have.

In the equations of Maxwell, there are two concepts for the electric and the magnetic fields called field strength and density. About those Maxwell said that "the first equation says that electric flux lines, if they end (my emphasis) at all, will do so in electric charges". The second states that "magnetic flux lines never terminate" (my emphasis). In an effort to re-interpret Maxwell conclusions, I went to a Merriam Webster dictionary of English and found that the definition of the word "end" is "the parts of an area that lies at a boundary; a point which marks the extent or limit of something or at which something ceases to exist; a ceasing of a course; the ultimate state". For "terminate" I found "to bring or come to an end; conclude, finish, or complete". From Maxwell and these definitions I drew an analogy between the word "terminate" with the concept of localization, whereas the 
phrase "never-terminate" with the concept of delocalization. This leads to the conclusion that the generation of an electric charge may be a consequence of localizing electric flux. Since magnetic flux lines are never localized, therefore, they are always delocalized. This could also be seen by saying that the electric charge is a consequence of spreading electric flux over a lower number of energy levels. Therefore, my hunch finds evidence in the fact that what the electric charge is missing is the ability to be delocalized or spread over a larger number of energy levels as in the case of the magnetic field. We can also hypothesize that the electric charge proceeds from a state of delocalization as the magnetic field, to account for a possible preference of spreading energy over higher number of energy levels, and thus giving a sense of supremacy or superiority to the delocalization state of the magnetic field.

If we consider a charged particle, from Maxwell and the above analogy, we may say that the charge proceeds from a quantum attenuation of the delocalization field responsible for terminating electric flux. We may speculate that the degree of localization is responsible for the size and the sign of the charge of the particle. This may explain how particles acquire charge: depending on the degree of attenuation in the localization of electric flux among lower number of energy levels, in which the degree of localization is controlled by the delocalization/localization quantum field. Likewise, we may apply the same reasoning to explain mass allocation in a particle. Let us introduce a violation to Maxwell second equation by allowing some extent of the magnetic flux lines to terminate or become localized by spreading its energy over a lower number of energy levels, as in the case of the electric flux lines. In this case we may hypothesize that the mass of the particle proceeds from a quantum attenuation of the delocalization field responsible for terminating magnetic flux. We may also speculate that the degree of localization is responsible for the amount of mass of the particle. In summary, the different electric and magnetic localized energy that builds our solid, material universe may proceed from a pure energy, wave-like matter source which energy may be $100 \%$ delocalized, $0 \%$ localized, and theoretically showing no duality or no particle-like properties. The model implies that particles may have different degrees of duality and that pure matter may have to move faster than what we call the speed of light to account for the larger spreading of energy over a larger or infinite number of energy levels. This kind of totally-delocalized energy can also be associated with the hypothetical particles called tachyons, which arise in quantum mechanics from a mathematical consideration of the expression for the energy and momentum given by special relativity (Feinberg, 1991).

In summary, mass and charge can be defined as the resulting observed vibrations from localized electromagnetic energy proceeding from a parent source or matrix that resembles the antique philosophical ether. It is worth to mention that Max Planck, 1918 Nobel Prize winner in physics and considered another of the fathers of quantum mechanics, mentioned that in reality there is no matter like atoms, that matter originates from a force that brings a particle into vibrations, and that behind this force exists an intelligent mind which he considered the matrix (my emphasis) of all matter (Planck, 1944). Some recent new ideas about the constant acceleration and expansion of the universe seem to correlate with the matrix concept presented here (Ruchvarger, 2011).

In quantum mechanics it is well known that the duality shown by all matter is a consequence of the wavefunction being composed by terms of both particle and wave, and hence the name duality (Granatstein, 1991). Quantum theory explains through the Heisenberg uncertainty principle that there are properties of particles than cannot be simultaneously determined, like the position and the velocity (Levine, 1991). In the HVB theory, this is explained by saying that there are two different kinds of fundamental energies, one is delocalized and the other localized. The difficulty of measuring both with precision arises from the fact that the delocalized portion is spread over a much higher number of energy levels than the localized. This includes the possibility that the delocalized energy must be evaluated based on speeds higher than the speed of light. There may be the possibility that the mathematical models that humans have used to measure energy are not entirely adequate to measure delocalized energy.

\section{The Tachyon-like Superluminal Matrix}

The tachyon-like matrix may be seen as the source from which superluminal matter has been slowed down to the speed of light (luminal) by localizing electromagnetic properties that allow building the fundamental particles of our human universe. The causation of the change from superluminal, or total delocalization, to luminal with attenuated degree of localization, is considered to be analogous to the Lorentz force associated to the electromagnetic force (McGraw-Hill, 1994). The driving force is associated to the concepts known as quantum coherence and decoherence, which are related to the natural ability and tendency of waves to get entangled to form conglomerates of localized energy. 
The Big Bang in the HVB theory is a result of a gigantic initial energy loss from a delocalized state into a localized state of lower energy, in which slowed-down energy waves started getting fast and violently entangled into shapes and motions resembling a vortex or a storm or a black hole. This finds an analogy in the description of the formation of the universe presented by the ancient Greek philosophers in times before Christ. For example, translated from Spanish, in the atomic-materialism philosophy, Leucipo describes the formation of worlds from "the separation of the infinite, in which multiple bodies of all kinds of species and shapes, which united, produced a vortex, in which they push and touch themselves, and move around in all ways" (Fullat, 1986). It may appear that once the vortex was started, the initial entanglement may have grown in size by attracting more newly-distorted matter through increased electromagnetic force, then moving into a state of stability or equilibrium under the new set of conditions, with little or no net flow of newly-distorted matter to or from the matrix source. The effect may give the macro-impression of being a self-created and isolated system having no observable net exchange of mass and heat with an external source. This may be responsible for giving the macro impression of energy conservation as described by the first law of thermodynamics. The process may be considered as noisy, exothermic, spontaneous, and the originator of the positive entropy of our universe as described by the $2^{\text {nd }}$ law of thermodynamics, the universal constant increase in disorder. Here, the initial loss of stability by the delocalized state is associated with going from a highly ordered state (higher stability) into one with increased disordered (lower stability). The theory contemplates the already-existing and not-well-understood suggestion that before the Big Bang all matter was ordered or compressed into a small point or dot of space. The HVB adds to the story that all the energy and mass of this universe were part of the originating matter, in which all electromagnetic energy appeared totally delocalized with zero charge and mass localization. Thus, the impression that cosmologists have about the compression or collapse of all the localized matter of the universe into a small dot of space is here complemented by a huge matrix universe where all the energy of the universe was originally delocalized. The HVB theory concludes that the formation of our universe is associated with an initial disorder in the matrix that gives rise to order on our universe, which in turns struggles to keep order because it lives under a condition of constant increased disorder. This implies that organizing objects in the universe goes against nature. Therefore it is logical to suspect that organizing something complex like the gene framework in DNA chromosomes that gives rise to life must have received a "non-natural" input of energy to reverse the disordering tendency. This energy may be associated with the delocalized energy of the tachyon-like matrix.

In summary, the HVB theory considers that our universe is made from distorted energy of the matrix capable of forming a "bubble" of solid matter that propagates at a slower speed indefinitely through the faster moving matrix. The model resembles a slow moving eddy on the surface of the rushing waters of the river. For the HVB theory, our universe is one eddy of the matrix. Therefore, the universe has a limited size and shape, perhaps curved because it started from a vortex of pre-localized energy of waves, which implies circular motion or internal rotation. This also implies that the universe must have an overall rotational angular momentum, mass, wavelength, and frequency most likely associated to the matrix where it comes from.

The HVB theory also utilizes the scientific fact that atoms are conglomerates of particles like quarks and electrons, and therefore are also waves, which vibrations are a fundamental concept of quantum mechanics calculations. This explains why the trigonometry sine wave function and its variations appear in the descriptions of the wavefunction of particles, and hence the description of atomic energy levels. Since our universe is made of atoms, therefore, because of the deBroglie relation, everything built from atoms, including humans, also vibrates through a very complex total summation or conglomerate of harmonic waves only "understood" through the mathematical procedure called Fourier transformation.

Humans can be seen as a complex well-organized arrangement of waves, a fact arising from the duality of the particles making humans. Humans live aware of the solid- localized energy of the universe, therefore, it should be logical to expect that whenever a quantum measurement is carried out, the best approximated measurement is the one coming from the localized energy. It should also be logical to expect that the wave nature of both the observing human and the instrumentation used to carry out the measurement may affect to certain extent the measurement of energy (Zinkernagel, 2011). An evidence to this and a confirmation to the Heisenberg uncertainty principle apparently comes from the double-slit experiment and the well-known problem of observer interference with the measurement of quantum states (Zinkernagel, 2011) in which the observation action of a human being and an instrument interferes with the natural state of the observed system. In the particular case of the light double-slit experiment the conclusion is that a photon behaves as a wave if it is not observed and as a particle if it is observed (Wolf, 1988). In my opinion, this is a consequence of everything in this universe being fundamentally made of energy waves, localized and delocalized. Indeed, the way in which atoms and sub-atomic 
particles are differed is by describing mathematically their vibrations. The total energy of an atom is a result of summing all the individual energies, such as electric, magnetic, kinetic, potential, etc. In atoms, the wavefunction is a sine-like mathematical equation that explains the electron vibrations based on quantum amendments to the concepts of the harmonic oscillator and the rigid rotor of classical physics (Levine, 1991). The model is based on the Newtonian oscillating pendulum motion, in which the energy of the allowed "curvy-like" vibrational oscillations is approximated through calculus operations. The wavefunction is really a representation of the infinite number of Planck-allowed energy values in which the particle can vibrate at different times. For example, under normal conditions (like $25^{\circ} \mathrm{C}$ temperature and 1 atmosphere of pressure), based on vibrations, each element of the periodic chart should have its own vibrational frequency or "signature" or "finger print", so that atoms of the element hydrogen $\left({ }^{1} \mathrm{H}\right)$, for example, can be distinguished from atoms of the element helium $\left({ }^{2} \mathrm{He}\right)$, and so on.

Therefore, it should be expected that anything built of molecules, as a result of combining atoms, must have a unique vibrational fingerprint. This is very well exemplified by genes in which every living organism including yeast, bacteria, viruses, plants and animals, in theory have a unique DNA make up (genome). In the case of humans, for example, there are in general two classifications of genes. One set is related to "house-keeping", which includes all genes common to all humans responsible for building a working human body. The other "unique" set is responsible for keeping every human different from any other human, therefore, assigning a unique individual genetic "fingerprint". Since genes are made of polymers of nucleic acids (Campbell, 1997), and nucleic acids are made of atoms, and atoms are particles, and particles are delocalized/localized dual vibrational waves, therefore, the different atomic structure that makes unique individual DNA is also responsible for generating a unique "finger print" overall vibrational frequency. Furthermore, including the whole animal and plant kingdoms, every DNA-based living organism should be expected to generate its own unique vibrational frequency. This wave (or sound) can be said to be "alive" or musical, because the DNA is subject to constant unfolding to carry the processes of replication, transcription, and translation (Campbell, 1997). Therefore, living organisms may be differed from non-living objects because objects only have a "standing" overall vibrational wave as a consequence of placing the atoms at defined locations based on chemical and symmetry rules.

Since humans can be seen as a complex well-organized arrangement of localized waves conglomerated into dual particles following a set of instructions carried by the genes in the DNA chromosomes (Campbell, 1997), therefore humans must also be energy propagated through a medium, even if the medium is "empty" or vacuum. The HVB theory hypothesizes that the energy matrix is the medium in which the energy of our localized universe is being propagated. As in all waves, the matter of the medium just serves to displace the energy with little or no net displacement of its own matter resembling the domino effect. The HVB theory proposes that the instruction to localize matrix energy into DNA molecules belongs to the superluminal energy of the universe that is propagated through the matrix. Using as an analogy the traditional "moving" light that goes around the sign of a movie theatre, the light bulbs of the sign represents the medium of the matrix, whereas the energy that turns on and off the lights in an orderly fashion is what carries the instructions of our universe. Notice that the universe is not being manifested in the glass of the bulb, which represents the superluminal matter of the matrix medium. The model suggest that it rather lives in the action denoted by the quantum absorption of energy that electrons experience in the tungsten $\left({ }^{74} \mathrm{~W}\right)$ filament, the effect responsible for the emission of light that illuminates the light bulb. The universe is present in a certain area of the matrix, that is, in a light bulb, for as long as the light bulb is turned on. The electrical instruction that turns on and off is the analogous to the instruction responsible for the manifestation of the universe in the emitted light of the piece of tungsten filament made of localized matter.

\section{Relation between Delocalization, Stability, and Energy Minimization}

As we have seen, the process of energy delocalization and localization is identified with the ability to spread energy over a larger or smaller number of energy levels. In the case of aromatic molecules such as the benzene ring, the energy of the $\pi$ electrons is spread around the hybrid molecular energy levels resulting from the overlapping of the atomic orbitals of the carbon atoms. In doing so, the molecule acquires stability and strength, because the spreading avoids electron-electron repulsions that would otherwise occur should the electrons were kept localized among a smaller number of carbon atoms. In the benzene ring, the electrons become delocalized by allowing them to move around the ring at high speeds, so that the real physical molecule is a hybrid that receives contribution from all the different positions in which the $\pi$ electrons can be. This is what is called in chemistry "resonance". The hybrid molecule has an energy value lower than any of the localized contributing structures, and thus structural stability is associated with energy minimization. The existence of the benzene ring 
suggests that in nature, localizing energy is equivalent to entrapment, leading to instability, and that, therefore, freedom and stability is achieved by delocalization. It is worth to mention here that, in theory, all molecules exhibit a certain degree of delocalization, suggesting that nature has a preference for energy minimization through delocalization.

\section{Mass and Charge as a Consequence of the Delocalization Quantum Field}

The initiating motivation of this work came by reinterpreting the first two electromagnetic equations of Maxwell. In quantum mechanics it is said that the electric and magnetic fields of a charged moving particle appear perpendicular to each other, that is, at a $90^{\circ}$ angle. My new proposal is that mass and charge proceed from attenuating the degree of localization of these fields, in the sense that they are forced to be entrapped by spreading their energy over a lower number of energy levels. This process increases duality, thereby giving each particle its own degree of duality. It is hypothesized that the two field vectors representing the electric and magnetic fields and/or electric and magnetic flux are not always separated by an angle of $90^{\circ}$.

Let us introduce a hypothetical moving particle with a mass and charge represented as a hybrid of a certain degree of localized electric and magnetic fields, consequence of an angle separation of their vectors from an initial state different from $90^{\circ}$. Let us pretend that the initial state has the vectors totally parallel to each other so that: 1) the particle has zero mass and charge when both the magnetic and the electric fields are parallel, equivalent to having all energy delocalized. 2) The particle has mass and charge different to zero when the vectors have an angle greater than zero, equivalent to having some degree of energy localization. In mathematical terms we may write:

$$
\mathrm{m}=0, \mathrm{E}_{\mathrm{f}}=0 \text { for } \mathrm{E}_{\mathrm{f}} \| \mathrm{B}_{\mathrm{f}}
$$

and

$$
m>0,\left|E_{f}\right|>0 \text { for } E_{f}\left\llcorner B_{f}\right.
$$

where $m$ is the mass, $E_{f}$ is the electric field, $B_{f}$ is the magnetic field, $\|$ is parallel or $0^{0}$ or aligned, and $L$ is any angle different from $0^{0}$ or unaligned. Since delocalization is associated with stability and localization with instability, we may also associate delocalization with greater order and localization with greater disorder, so the entropy (S) of the particle is increased for all $E\llcorner B$ degrees of separation. Thus, the change in entropy $(\Delta S)$ for going from $\|$ to $L$ is positive. Since localization has lower number of energy levels than delocalization, then the energy change $(\Delta \mathrm{E})$ is:

$$
\Delta \mathrm{E}=\mathrm{E}_{\text {final }}-\mathrm{E}_{\text {initial }}=\mathrm{E}_{\text {localized }}-\mathrm{E}_{\text {delocalized }}=\text { Number with negative sign. }
$$

If $\Delta \mathrm{E}$ can be associated with enthalpy $(\mathrm{H})$, then we may say that the process is exothermic, in which energy in the form of heat is released whenever localization occurs from a previous state of delocalization. Since $\Delta \mathrm{S}$ is positive and $\Delta \mathrm{H}$ is negative, therefore the process of going from $\|$ to $L$ is spontaneous, and the reverse or going from $L$ to $\|$ is non-spontaneous and endothermic. This means that the reverse process of going from localized energy back into delocalized requires inputs of energy, and if too large, the process may actually be irreversible, at least under normal conditions.

\section{A Lorentz-Like Force May Cause the Split of the Vectors}

I have proposed that the mass and charge of particles is a consequence of the degree of separation between the two vectors of the electric and the magnetic fields/flux from an initial aligned state (II) into a final unaligned state $(\llcorner)$. There are three immediate questions arising: 1) what is the meaning of the initial alignment state? 2) What causes the separation, split, or misalignment? 3) What is the difference in speed when particles go from delocalized to localized? Let us define the initial state in which the field vectors appear parallel (II) as a matrix composed of a matter having properties such as electric and magnetic totally delocalized or spread over an infinite number of energy levels. Let us call this kind of matter "pure energy". Assuming that a force similar to the electromagnetic Lorentz force is responsible for localizing energy of the matrix matter, and since $\Delta \mathrm{E}$ is negative, therefore, the parent matrix of pure matter should experience a loss of kinetic energy in order to account for a slow-down from superluminal or tachyon-like speed. Since the whole process is exothermic, it can be assumed that it is very hot and extremely noisy, very similar to what is theorized about the Big Bang.

\section{Time}

In the process of energy localization from an initial state of delocalization due to the action of a Lorentz-like force, what physicists call time can be seen as one resultant quanta of the delocalization field, and thus time can be considered another waveparticle having duality, that is, a hybrid having properties of both particles (mass and momentum) and waves (wavelength and frequency). The idea that time and mass should not be treated 
differently in terms of quantum mechanics has been mentioned before (Hilgevoord, 2005). In physics, mass and momentum are identified with localization, whereas wavelength $(\lambda)$ and frequency $(v)$ with delocalization. Let us assume that time is another duality phenomenon in which the wave property is much larger than the particle property, that is, having more delocalized energy than localized, in which the localization of pure energy is responsible for providing certain quantum degree of duality to the waveparticle time.

To explain how time behaves, we may consider that: 1) time may be associated with a unidirectional flow gradient. 2) Time is associated with certain quantum energy states or levels, consequence of the distortion or slow-down of the matrix pure energy. Here, time can be seen as stages in which particles with higher degree of localization or solid-like properties, such as quarks and electrons, can be manifested. In this model, this localized matter is the analogous of the tungsten filament in the light bulb, whereas time is the analogous of the light emitted by the excited electrons where the universe is manifested. 3) The unidirectional flow of the force controlling the time gradient is associated with a negative change in energy $\Delta \mathrm{E}$, so that every event occurs at a different energy level of time. This would set any event 1 , or the cause, at a higher energy level than the subsequent event 2 , or the consequence. This would allow proper ordering of all events in a time sequence, all based on a Planck-like $\Delta \mathrm{E}$ difference. 4) Since every two consecutive events lead to a negative $\Delta \mathrm{E}$, therefore, traveling back in time from the consequence to the cause is forbidden, because the Planck-like energy needed to reverse the natural exothermic and increased-entropy may not be available in this universe, rendering the process irreversible. 5) On the other hand, traveling forward into the future is exothermic, spontaneous, and most likely restricted to $\Delta \mathrm{E}$ changes between consecutive cause and effect events. One immediate consequence of these limitations is that, as the universe propagates, event 2 , or the consequence has lower time-like energy available to recreate it than any previous event 1 . We may, unfortunately, conclude that as the universe expands from the Big Bang, it gets continuously replaced with less availability of time-energy to recreate it with a slight increase in disorder. It also suggests that running out of time-like energy to recreate the universe is analogous to bursting the bubble of localized energy that makes this universe.

\section{Energy Minimization and the Proposed $4^{\text {th }}$ Law of Thermodynamics}

In the hypothesis in which every event in this universe occurs at a different energy level of the waveparticle time so that $\Delta \mathrm{E}$ is negative, it was concluded that any new event has lower energy available to be recreated with further increased in entropy. This concept can be associated with the ubiquitous universal law of minimizing energy use implicitly present in the science fields of physics, chemistry and biology. For example, in the benzene ring, energy delocalization through resonance has the consequence of creating a hybrid structure in which energy from electron repulsion is minimized, so that the resulting structure is stable or energetically-favored under normal conditions. In science classes students are taught about many examples in which this natural tendency is sustained. Scientists are aware of this phenomenon despite of its implicit existence behind the laws of thermodynamics. It is very possible to cite a large number of examples to make the point of view stronger, however, I would like to limit to a few that demonstrate that the pattern repeats at higher levels of organization. In organic chemistry, there are many reactions that could be explained through several molecular mechanisms or pathways, however, nature prefers the one occurring through lower energy expenditure. In behavioral biology, animals prefer to make a threatening noise or fly away rather than spend lots of energy by engaging into fight. In human society, energy use is always minimized, especially when energy is translated into money. Since energy is tried to be minimized at all causes, regardless of the scale, then it is fair to claim that there should be an amendment to thermodynamics by calling the $4^{\text {th }}$ law as follows: During any process, energy is always minimized.

Calling this implicit law an official law, would probably help moving forward from the little advancement that physics have seen since the contributions of past great scientists. For example, it would be possible to dismiss bizarre theories such as the parallel universes, which boldly extrapolates the duality of the wavefunction of particles into human existence (Wolf, 1988). In the parallel universe theory, there are versions of you and me going on with the lives consequence of the decisions that we did not choose in our universe, in which new universes sprout every time we take a particular choice. Based in this theory, the decision of undergoing heart beats to keep you alive in this universe has a counterpart parallel universe in which the decision is not undergoing heart beats, so that in that parallel universe you are dead. However, if energy has to be minimized, then creating universes every now and then to represent the lives of other versions, including the one dead, can be considered a reckless and forbidden expenditure of energy and thus a violation to the $4^{\text {th }}$ law.

The HVB theory shares with the parallel universes theory that the duality of matter is a consequence of the quantum wavefunction. In both theories, the universes of our other versions are represented by distinctive wavefunctions. The total number of wavefunctions, or possibilities for a new outcome, tends to infinity because 
of the superpositions of individual harmonic wavefunctions called linear combinations of harmonic oscillations. In the HVB theory the waveforms of possibilities really exist in the delocalized world, representing the ways in which the matter of the matrix can be distorted into an outcome of an event. All is happening inside the time waveparticle energy level in which a particular event is manifesting. At the moment of taking a decision, all allowable waveforms are available to represent each possible outcome. Here, the selection for a waveform that represents a particular decision is carried out by another component of the superluminal energy identified with human consciousness and its freedom of choice. Whenever a decision is taken, all possible delocalized wavefunctions collapse into the wavefunction that represents the observable of the localized outcome. Once the outcome is consumed, a new lower time energy level is in charge of providing the set of delocalized wavefunctions that represent the new possibilities allowed for the next possible outcome, everything subjected to the condition of lower energy availability. In this way, energy is minimized and not recklessly wasted by multiplying the universe every now and then. In summary, all possible outcomes exist only in the delocalized universe before the decision is taken, whereas the chosen only exists as a hybrid of all choices in the localized universe, so that there is only one solid universe determining our present "reality".

\section{Quantum of the Delocalization Field}

Previously it was mentioned that the delocalization/localization field is associated to an energy that carries a quantum instruction of how to distort or bend the propagation matrix to shape the universe at a time $t$. Here, time is considered a dual waveparticle and a resulting quantum of the distortion field, defined as the smallest quantity or packet of energy associated with the recreation of the universe in the matrix. Also it was clarified that an event is a particular shape in which the matrix is bent and that successive events of cause and effect occur at decreasing levels of time-like energy, so that the cause occurs at a higher energy level than the effect. The relation between time and events is that the waveparticle time represents the stage at which every event occurs, implying that time determines the amount of delocalized matrix energy that can be localized to build our solid universe with certain degree of entropy. If placed in a spectrum of quantized duality, time may appear as a parent waveparticle of all waveparticles, made of, for example, $99.99 \%$ delocalized energy and $0.01 \%$ localized energy, thereby showing a very low percent of duality. Further localization of energy can make heavier waveparticles such as light, quarks, electrons, and the rest of the particle plethora. The function of a time parent waveparticle could be analogous to the role of the biological stem cells. All waveparticles result from different quantum attenuation of duality, which overall effect determines the degrees of matrix distortion. Mass and charge are like quantized energy packets of linear combinations of localized magnetic and electric fields and/or flux. In this way, both mass and charge may be treated as light, in other words, being made of electromagnetic waves consisting of oscillating electric and magnetic fields. Each possibility for the energy is determined by the degree of duality, represented by a wavefunction $\left(\Psi_{\text {dual }}\right)$ that receives contribution from both delocalized and localized energy:

$$
\Psi_{\text {dual }}=\Psi_{\text {localized }+} \Psi_{\text {delocalized }}
$$

In the equation, $\Psi_{\text {localized }}$ corresponds to a vectorial wavefunction receiving contribution mostly from the localized energy responsible for generating the solid-like traits or characteristics of matter such as mass and momentum, whereas $\Psi_{\text {delocalized }}$ receives most contribution from the delocalized energy responsible for wave-like traits such as wavelength and frequency. In other words, $\Psi_{\text {localized }}$ is the energy of the vortex, bubble, distortion or eddy that shapes this universe, whereas $\Psi_{\text {delocalized }}$ is more matrix-like. To account for the theory of relativity, therefore, $\Psi_{\text {localized }}$ must be defined in terms of the luminal speed of light, whereas, $\Psi_{\text {delocalized }}$ must be defined in terms of the superluminal tachyon-like speed. $\Psi_{\text {dual }}$ at a particular time $t$ may itself be defined by linear combinations of $\Psi_{\text {localized }}$ and $\Psi_{\text {delocalized, }}$, where each $\Psi_{\text {localized }}$ and $\Psi_{\text {delocalized }}$ is also defined by linear combinations of individual $\psi_{\text {sub-levels: }}$ :

$$
\Psi_{\text {dual }}=\sum \Psi(\psi)_{\text {localized }}+\sum \Psi(\psi)_{\text {delocalized }}
$$

To represent the degree of distortion or duality, I have drawn an analogy with the $90^{\circ}$ angle that physics professes to be between the electric and magnetic fields of all moving charged particles. A thorough search in the literature to corroborate that all moving charged particles generate a magnetic field exactly at a $90^{\circ}$ angle has produced little information, in particular, studies regarding magnetic fields generated by particles with substantially different mass. In this model, I violate this claim to speculate that these two fields are not exactly at a $90^{\circ}$ angle for all kind of particles, so that the degree of duality can be represented by changing the angle from perpendicular to obtuse or acute. In this case, a particular $\Psi_{\text {dual }}$ wavefunction defines certain angle between the $\Psi_{\text {localized }}$ and $\Psi_{\text {delocalized }}$ vectors responsible for the waveparticle properties at a time $t$. 
Let us assume that all luminal particles acquire stability whenever linear combinations of $\Psi_{\text {dual }}$ give rise to a $90^{\circ}$ angle between the magnetic (B) and the electric fields (E), as established by physics. Since duality can be scrambled, we can say that certain linear combinations are associated with a ground state, determined by the average behavior of the particular type of particle under normal conditions. The ground state could be obtained from linearly-combined $\Psi_{\text {localized }}$ and $\Psi_{\text {delocalized }}$ wavefunctions that lead to a stable conformation of the particle that ensures a $90^{\circ}$ angle between $\mathrm{E}$ and $\mathrm{B}$. In contrast, other combinations may represent statistical deviations from $90^{\circ}$ that lead to an acceptable less stable conformation, while others may lead to totally unstable forms of the particle. The conclusion is that each particle has the opportunity to be built in different ways from a "pool" of possible linear combinations of $\Psi_{\text {localized }}$ and $\Psi_{\text {delocalized }}$ wavefunctions allowed for its kind. Utilizing this concept, the mass, charge, and type of any particle can be described through different attenuation of duality. For example, a photon would have: 1) mass defined from the amount of localization of magnetic energy, from the previously proposed violation to Maxwell equation \#2. 2) Charge defined from the amount of localization of electric energy, as given by Maxwell's first equation. 3) Type or flavor defined from differential linear combination of individual $\Psi_{\text {localized }}$ and $\Psi_{\text {delocalized }}$, 4) $\Psi_{\text {localized }}$ based on luminal speed and $\Psi_{\text {delocalized }}$ based on superluminal speed. In this case, we can say that the red, green, and blue (RGB) photons, for example, have different wavelength and frequency because they have different duality given by a $\Psi_{\text {dual }}$ determined by different degrees of $\Psi_{\text {localized }}$ and $\Psi_{\text {delocalized }}$ linear combinations. Each photon determining a color of the light may have a unique dual wavefunction $\Psi_{\text {dual }}$ coming from a pool of linear combinations of localized $\left(\psi_{\text {loc }}\right)$ and delocalized $\left(\psi_{\text {deloc }}\right)$ wavefunctions characteristic of photons. Since photons have certain amount of localized energy $\Psi_{\text {localized, }}$, therefore, they must have a mass different from zero, although extremely low because $\Psi_{\text {delocalized }}$ could be significantly bigger than $\Psi_{\text {localized }}$ because light may be, for example, $99 \%$ delocalized, $1 \%$ localized. Indeed, the fundamental concept of duality of quantum mechanics is based on the description of the photon as a waveparticle. The mathematical treatment works to predict the behavior of light assuming that the photon has zero mass, although there has been a sense of doubt in the scientific community (Levine, 1991).

Like light, if time is considered a dual waveparticle, therefore, time would also have mass, though significantly smaller than light because the $\Psi_{\text {localized }}$ of time would be significantly smaller than the $\Psi_{\text {localized }}$ of photons. Therefore it could be said that time, as compared to light, is less dual, and behaves more like a wave than like a particle. In general, the different flavors in particles, such as those in quarks, can also be explained through attenuations of duality by saying that each flavor has its own set of linear combinations of $\Psi_{\text {localized }}$ and $\Psi_{\text {delocalized }}$ giving rise to a particular mass, charge, spin, momentum, frequency, wavelength, etc. In the same sense, we can also say that heavier particles such as electrons, protons, neutrons and atoms behave more like a particle than a like wave. Heavier particles would be more dual by having a value for the $\Psi_{\text {localized }}$ bigger than photons, electrons, and quarks. These therefore are more able to show a trait consequence of larger energy localization such as the observed macro-solidity of the substances made by clusters of atoms such as molecules, cells, living organisms, and the rest of the objects of the universe.

The difference in the flavors of quarks can be explained by assuming that the wavefunction $\Psi_{\text {dual }}$ of a particular flavor depends on a particular duality given by linear combinations of localized $\left(\psi_{\text {loc }}\right)$ and delocalized $\left(\psi_{\text {deloc }}\right)$ energy obtained from a pool of $\Psi_{\text {dual }}$ S characteristic of quarks. The $\Psi_{\text {quarkflavor }}$ function for a particular quark would have its mass given by the amount of localized magnetic energy (violation to Maxwell's second equation), charge from the amount of localized electric energy (Maxwell's first equation). Likewise, the electron, proton and neutron have the mass and charge given in a similar fashion, however, antiparticles (Dirac, 1931) may introduce a different effect. Since the $\Psi$ functions for a particle and its anti-particle have same Maxwell-violation mass and same Maxwell charge but different sign, it is postulated that the difference in the sign is due to a change in the orientation between the magnetic and electric fields/flux. For example, one orientation $\left(90^{\circ}\right)$ may favor the observed negative charge in the particle, while another, for example $-90^{\circ}$, may favor the observed positive charge of the antiparticle.

Isotopes may also have a particular linear combination of duality that changes the behavior of observed traits like radioactive decay. For a particular element, the identity of the element is kept by the amount of localized energy assigned to protons and electrons, however, the differential energy localized in neutrons changes the duality of the atom. The change can be represented by a $\Psi_{\text {dual }}$ function obtained as a consequence of a substantial deviation of the electric and magnetic fields from the stable $90^{\circ}$ angle, leading to an unstable but acceptable form of the atom. Since duality is quantized, therefore we may say that: 1) certain linear combinations of localized/delocalized energy define the average dual stability of the most stable form of the isotope. 2) Other certain linear combinations lead to deviations that are still acceptable to build a version of the isotope, however, at the expense of losing the ability of retaining neutrons in the nucleus. In the case of isotopes, each $\Psi_{\text {dual }}$ 
representing different accep table d eviations from the perpendicularity may be as sociated to different $r$ ates of radioactive decay. Indeed, this would solve Einstein's quote "God does not place dice with the universe". For a summary see Figure 1.

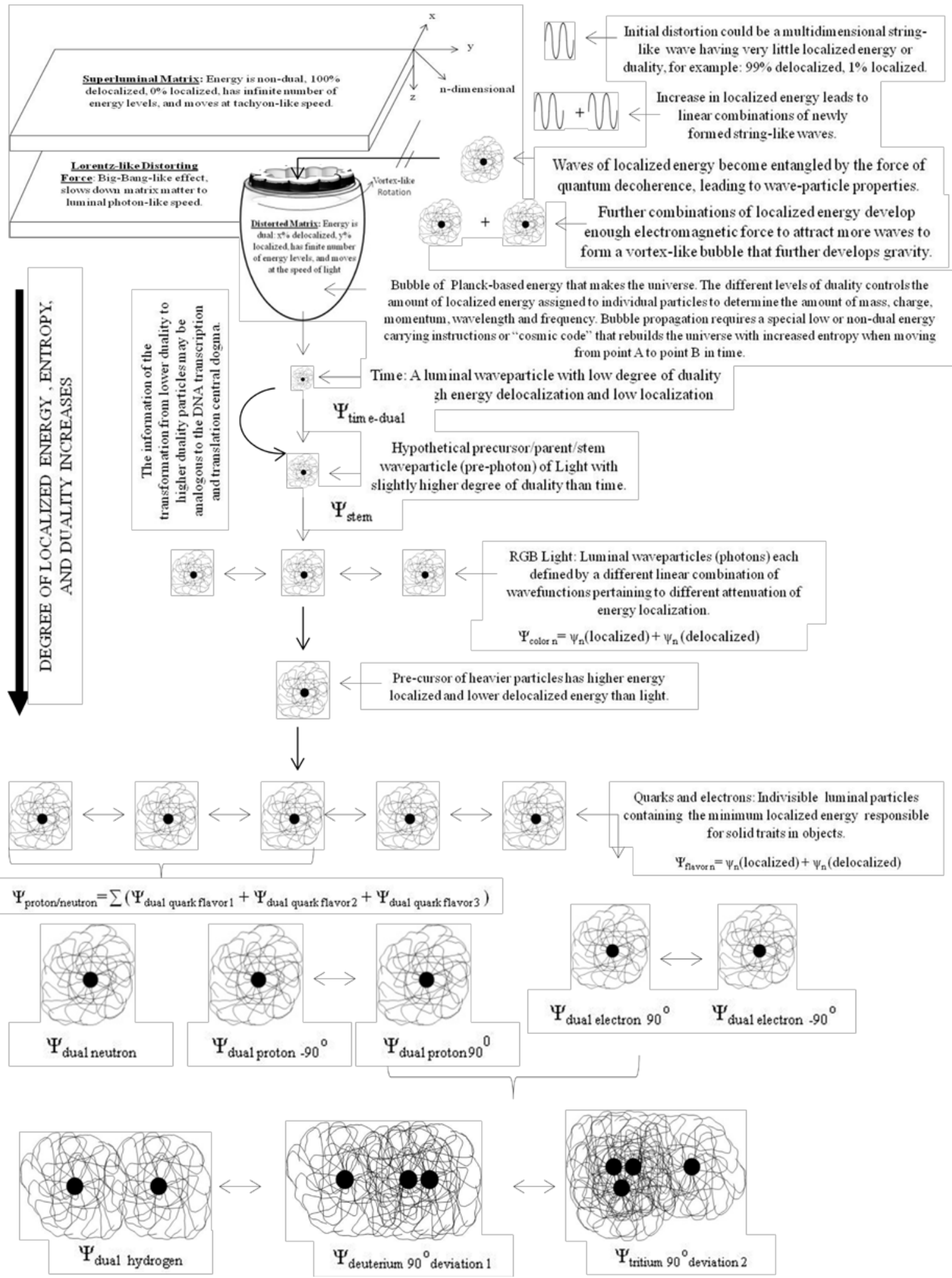

Figure 1. Summary of the origins of the particle plethora based on the HVB theory 


\section{A Brief Look at Evolution}

In the HVB theory, the problem of the chicken and the egg gets re-studied to reinterpret the theory of evolution. In the same way that this paradigm found an apparent solution in genes by suggesting that none of them came first but the instructions stored in genes to build both the chicken and the egg, the theory of e volution can be amended by considering a new concept that I call the sand box. In the sand box theory, the matrix provides all the localized particles to build organisms. With different instructions obtained from the delocalized universe you can use the same localized materials to build different organisms independently and not necessarily through a mutually-connected lineal evolution. In this way, primates are not necessarily the ancestors of humans. They just share the same ingredients provided by the sand box.

\section{Conclusion}

Throughout hum an history philosophers a nd $\mathrm{s}$ cientists have gone t hrough de ep $\mathrm{t}$ houghts a nd de tailed experiments a ttempting to explain the na tural $p$ henomenon of 1 ife. The most outstanding $p$ roposals a bout the origins of matter, life, and human consciousness are very complex to comprehend. The main obstacle is the fact that the reasoning about origins requires collecting a huge amount of knowledge from across all fields of study to be able to create a logical story that $\mathrm{c}$ an explain life be yond doubt (Richardson, 2003). One major obstacle is posed by modern physics, which appears unable to clarify fundamental concepts such as mass and time.

The hidden variable behind philosophy may be a co nsequence of humans ap pearing on earth after a very huge and unknown number of cascade-like effects that created this universe. Our conscious minds became aware of what we cal led " reality" af ter many p rocesses were i nitially s et a nd p roperly $r$ unning in what we cal $l \mathrm{the}$ universe. Whenever scientists speculate that they have come to a final end of a story, it happens that in the future some other things cannot be ex plained and/or complicate the matter by opening new questions. A prominent physicist of the current $21^{\text {st }}$ century has very recently suggested in a publication that the universe made itself and that it was not created by God (Gabbatt, 2010). Proposals of that sort appear to be more personal than scientific, perhaps $g$ enerated $f$ rom the illusive $a$ nd $b$ linding $i$ dea o $r$ eaching a s tate of ab solute $k$ nowledge, $p$ erhaps proceeding as a result of ignoring the ever-present effect of discovery and re-discovery responsible for clarifying and o pening $n$ ew $q$ uestions. In co ntrast, $o$ ther $s$ cientists $h$ ave $p$ ostulated that there will al ways $b$ e a hidden variable that humans couldn't measure, which would otherwise clarify some things and/or complicate others. It always appears that human scientific research never finds an absolute end, regardless of the target concept. This may be a consequence of humans being smaller than the entire system, so that the ability to comprehend the system becomes greatly impaired.

New discovery of hidden variables is evidence that our minds became aware of reality after certain fundamental settings were in place. This may imply that we may need to de-construct so much to be able to observe the true initial origin of life, a process similar to reversing the cascade effect that continues to occur after the Big Bang. A f ew very i mportant q uestions ar e: 1 ) ar e we cap able of $d$ iscovering al $1 \mathrm{p}$ rocesses that cas caded through evolution giving rise to us and our reality? 2) At which point of the cascade we became conscious? 3) What if many of those cascading processes are so subtle that are close to impossible to be detected by our instruments or conscious minds? 4) Could it be possible that at this level of subtleness is where an outside intelligence (like who we call God, for example) truly operates through mechanisms similar to the butterfly effect?

Even in the case of collecting truly representative data that best approximates the answers to these questions, there is a possibility of failing to explain life correctly. An example is chaos theory, which leads into a false conclusion, even from the mental illusion of never-ending amounts of knowledge. We also have to realize that all human thoughts and experiments are subject to the increased disorder posed by positive entropy. From natural rules, I $\mathrm{c}$ an infer that if an individual does not supply the right a mount of energy ne eded to organize thoughts into clarity, then the individual is supposed to finish disordered and confused. Therefore, it could be concluded that it is naturally easier to get confused than clarified. In other words, getting clarified and organized requires negative e ntropy, which is a process that go es a gainst the flow of nature, and he nce requires s pending more energy perhaps expressed in the form of knowledge, effort and dedication. From the HVB point of view, we may say that extreme thoughts such as the a theist denial of the existence of an intelligent mind are consequence of following the $\mathrm{n}$ atural flow of pos itive e ntropy, in which the individual continues to be more confused than clarified.

The ph ilosophy of s cience s hould be ba sed on s peculation a nd $\mathrm{r}$ e-consideration of "well-established" fundaments. Perhaps the most remarkable contribution to the HVB theory is the proposal of the quantization of duality $\mathrm{r}$ esulting $\mathrm{f}$ rom $\mathrm{t}$ he a ttenuation of $\mathrm{t}$ he $\mathrm{d}$ egree o $\mathrm{f} d$ elocalized an $\mathrm{d} l$ ocalized energy a llocated i $\mathrm{n}$ a waveparticle. In summary, we may conclude that there are three main categories or entities: 1) the physical body 
composed of solid localized energy. 2) The "spiritual" body, composed of a subtle delocalized energy. 3) The rest o f t he universe, which as f ar a s humans know, co nsists o nly of $\mathrm{p}$ hysical an $\mathrm{d} \mathrm{c}$ hemical $\mathrm{r}$ eactions a nd equilibriums. The physical body and the rest of the universe are made from the slowed-down particles of the superluminal matrix. Solid particles are formed because the matrix matter becomes distorted/disturbed/bent from its normal state by a set of instructions that encode one way in which it can be slowed-down and distorted. For our universe, we may say that the Planck constant that regulates particle behavior is itself one quantum number belonging to a mode in which matrix matter can be distorted, see Figure 2. The different modes of distortion represent the several ways or levels of disorder, and thus accounting for a quantization of entropy, which would determine the al lowed an $\mathrm{d}$ forbidden distortions, $\mathrm{s}$ ee $\mathrm{F}$ igure 2 . This i ntroduces the $\mathrm{i}$ dea that there $\mathrm{c}$ ould be universes in which entropy is reversed or negative, so that everything, by nature, would have a constant tendency to $g$ et ordered. In my o pinion, $\mathrm{b}$ ased o $\mathrm{t}$ his co ncept, $\mathrm{p}$ erfection may $\mathrm{b}$ e d efined as the et ernal ab ility a nd tendency of a system to get ordered.

Harmonic oscillator model of the quantization of duality. Showing distinct vibrational "bubbles", each having unique speeds of light associated to unique Planck-like constant and wavefunction $\Psi_{\text {dual }}$ given by linear combinations of $\psi_{\text {delocalized }}$ and $\psi_{\text {localized. }}$

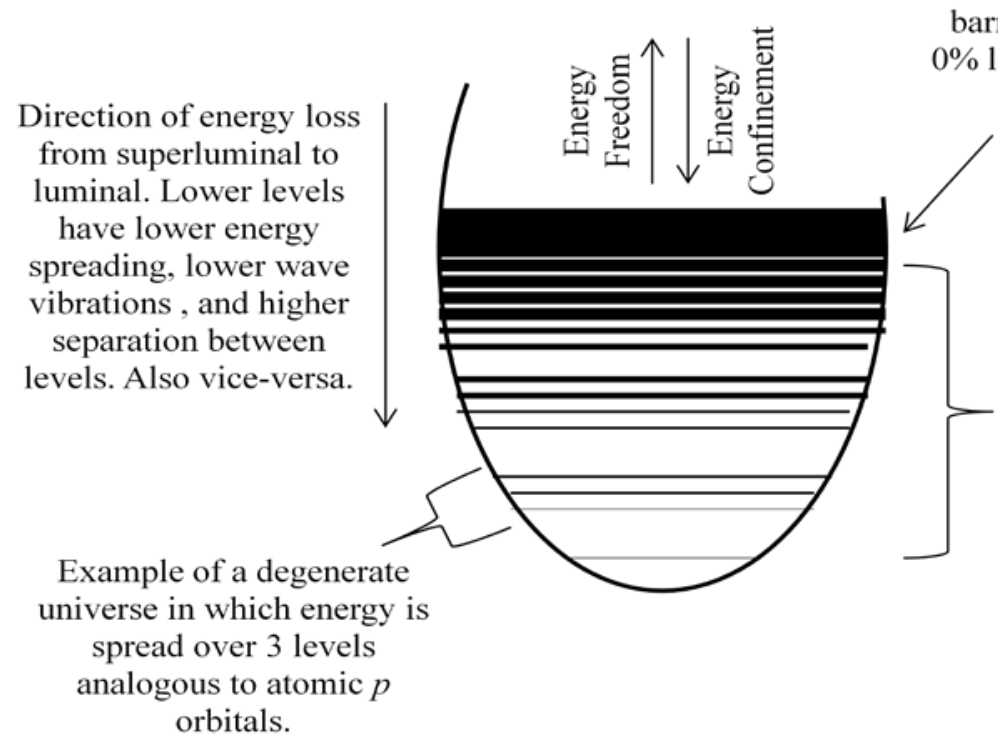

Continuum: Matrix matter of pure superluminal wave vibrations having lowest wavelength and highest frequency. Multi-dimensional universe, no energy barriers, no duality, $100 \%$ delocalized, $0 \%$ localized, where energy is allowed to be at all levels simultaneously.

Energy barrier separations between universes. Each universe has unique initial set of duality determined by $\%$ of energy delocalized and \% localized.

Figure 2. Harmonic oscillator model of the quantization of duality

Each energy level represents a universe having its own Planck-like fundamental constant. Each universe has its own set of instructions and al lowable vibrational frequencies for building objects and organisms, implying that each universe has its own periodic chart. The vibrational energy and the ability of spreading energy a mong a larger number of energy levels in creases from bottom to to $p$, so th the most stable universes exist in higher levels of e nergy d elocalization. $T$ his is possible b ecause in creasing e nergy is p roportional to the division of universes into larger number of sub-universes. This is known as degeneracy. Notice that the higher the vibration, the smaller the energy barrier between universes. The continuous area shown at the top represents the largest level of delocalization, in which there is no energy barrier between universes. In the continuum, the energy is like be ing spread over an infinite number of universes at the same time. As a n example, o ur 3 -dimensional universe can be represented by an $p$-like atomic orbital, in which the energy is simultaneously spread over the projections $p_{x}, p_{y}$, and $p_{z}$. Our universe may vibrate faster that a lower s-like universe (1-dimensional), however, slower than a $d$ (5-Dimensional) or $f$-like (7-dimensional) universe. If our universe resembles a $p$-like atomic orbital, then the bubble that it makes in the matrix should have a lobular shape.

The matrix matter can be bent in different modes perhaps by instructions or programs written in an electromagnetic-kind of language that tells how to change certain properties that controls its behavior. Just like 
genes, which encode instructions for life, there may be an analogous electromagnetic entity that encodes the instructions for any particular distortion. I propose the name matrixenes: gene-like superluminal electromagnetic instructions carried by delocalized energy that encodes how to re-build the universe every time it propagates through the matrix from point $A$ to point $B$ along its eternal accelerated expansion.

Notice the fact that, if humans and the entire universe are made of localized matter from the matrix, represented as waves of a slower-moving eddy in the faster-moving river, or as the light that illuminates a bulb in the theater sign, then they both are subject of being re-built or replaced by distorting the matter of the matrix present at the particular point $A$ or $B$. This implies that every time the universe propagates in its eternal expansion, it needs to be constantly replaced by different matrix matter, with the condition of rebuilding it with an increased degree of disorder. This concept is analogous to the biological fact that humans and all living organisms are constantly replacing the cells of the body, with the condition of becoming older. The replacement mechanism may operate at a time and error scale that our human brains cannot detect. An analogy is the failure of our brains to capture the divisions between frames in moving pictures. In this way, the HVB theory suggests that the real human essence is more associated with the "spiritual" subtle delocalized superluminal energy being propagated and replaced rather than with the solid localized matter that the matrix lends us to house our "spirit". The replacement concept can be also used to argue against the theoretical traveling in time. Notice that if energy use is minimized, then we can assume that there is one set of instructions needed to rebuild the universe at a point in the matrix. This means that when the universe propagates from point $A$, to point $B$, to point $C$, and so on, the instruction has been initially moved to point $B$ from point $A$, so that there is no instruction left over in point $A$ to leave a permanent copy of the solid universe as it was. Likewise, there is no solid universe ahead in point $C$ because the instructions of making the solid universe are still in point $B$. In conclusion, as stated before, there is no traveling in time because the only solid universe is the one in the present time.

In genes the force that drives the instructions is the electromagnetic force, which allows atoms and other particles to interact dynamically by nature in a particular way, consequence of the particular set of instructions. The electromagnetic force is considered natural/intrinsic, and thus, a property that depends on changes in the original instruction or program. What we perceive as "reality" is a result of the initial instructions that change the properties of the particles of the matrix, and thus the way that they behave. You can change "reality" by changing the instructions of the program that bends matrix matter to a Planck-like quantum value representing a fundamental instruction of a different universe. In other words, the matter of the matrix can be controlled/manipulated/distorted/bent, perhaps at will, to take a particular shape, whether the intention is for good or for evil. One may require higher effort than the other. I'm afraid that the option "for good" is the hardest. It may be true that it is easier to distort "for evil" than distort "for good". Perhaps the best option is distort not at all because distorting involves instability and greater disorder within the built system. We may speculate about why and how human energy became localized into an eddy or light-bulb-like form of universe slowly moving in a matrix of rushing waters or fast flicking of the on/off electricity switch. One aspect is for sure, we may all be physically made from localized energy of matrix matter but our true reality may still mysteriously be within the laws of superluminal physics, which go beyond our current understanding. My conclusive philosophical quote is that if the matrix is God, then we are all made from parts of God living in a universe inside of God. Since the matter of God has been distorted to allow us to live the way we do, then what we perceive as reality is not really the reality.)

\section{References}

Baez, John C. (2006). Open Questions in Physics. Retrieved from http://math.ucr.edu/home/baez/physics/General/open_questions.html

Bartelsa, A., Lyrea, H., \& Esfeldb, M. (2004). Holism in the philosophy of physics: an introduction. Studies In $\begin{array}{lllll}\text { History and Philosophy of Modern Physics, } & \text { 35, }\end{array}$ http://0-dx.doi.org.lib.utep.edu/10.1016/j.shpsb.2004.09.001

Bell, John S. (1964). On the Einstein, Podolsky Rosen Paradox. Physics. 1, 195-200. http://www.drchinese.com/David/Bell.pdf

Bohm, David. (1957). Causality and Chance in Modern Physics. London: Routeledge \& Kegan Paul. http://dx.doi.org/10.4324/9780203201107

Bohm, David. (1980). Wholeness and the Implicate Order. New York: Routledge, (Chapter 4: The Hidden Variable in the Quantum Theory). 
Bradley, Dowden. (2001). Time. Internet Encyclopedia of Philosophy. Retrieved from http://www.iep.utm.edu/time/

Brooks, Michael. (2005). 13 Things That Do Not Make Sense. New Scientist. Retrieved from http://www.newscientist.com/article/mg18524911.600-13-things-that-do-not-make-sense.html

Burnham, Douglas. (2001). Space, Time, and Indiscernibles. The Internet Encyclopedia of Philosophy. Retrieved from http://www.iep.utm.edu/leib-met/\#H7

Butterfield, J., \& Pagonis, C. (2002). More than the conscience of physics? From physics to philosophy. Studies In History and Philosophy of Science Part B: Studies In History and Philosophy of Modern Physics. 33(3). 576-582. http://dx.doi.org/10.1016/S1355-2198(02)00025-4

Callender, Craig, \& Huggett, Nick. (2004). Physics meets philosophy at the planck scale: contemporary theories in quantum gravity. Studies In History and Philosophy of Science Part B: Studies In History and Philosophy of Modern Physics, 35(3). 531-537. http://dx.doi.org/10.1016/j.shpsb.2004.04.002

Campbell, Neils A, Mitchell, Lawrence G., \& Reece, James B. (1997). Biology Concepts \& Connections (2 $^{\text {nd }}$ Ed.). California: The Benjamin/Cummins Publishing Company.

Carrol, Sean. (2012). Sean Carrolls research webpage. Retrieved from http://preposterousuniverse.com/research/

Chimisso, Cristina. (2008). From phenomenology to phenomenotechnique: the role of early twentieth-century physics in Gaston Bachelard's philosophy. Studies in History and Philosophy of Science, 39, 384-392. http://dx.doi.org/10.1016/j.shpsa.2008.06.010

d'Agostino, Salvo. (2003). A history of the ideas of theoretical physics: Essays on the 19th and 20th century physics. Studies In History and Philosophy of Science Part B: Studies In History and Philosophy of Modern Physics, 34(4). 677-679. http://dx.doi.org/10.1016/S1355-2198(03)00068-6

d'Espagnat, Bernard, \& Esfeld, Michael. (2006). On Physics and Philosophy. Studies In History and Philosophy of Science Part B: Studies in History and Philosophy of Modern Physics, 38(4), 989-992. http://0-dx.doi.org.lib.utep.edu/10.1016/j.shpsb.2007.05.001

Dirac, P. A. M. (1931). Quantised Singularities in the Electromagnetic Field. Proceedings of the Royal Society A, 133, 60. http://dx.doi.org/10.1098/rspa.1931.0130

Dürr, D., Goldstein, S., \& Zanghi, N. (1995). Quantum Physics Without Quantum Philosophy. Studies in History $\begin{array}{lllll}\text { and Philosophy of Modern } & \text { 137-149. }\end{array}$ http://0-dx.doi.org.lib.utep.edu/10.1016/1355-2198(95)00009-7

Einstein, A, Podolsky, B., \& Rosen, N. (1935). Can quantum-mechanical description of physical reality be considered complete? Physical Review, 47. 777-780. http://dx.doi.org/10.1103/PhysRev.47.777

Feinberg, G. (1991). Encyclopedia of Physics ( $2^{\text {nd }}$ Ed.). New York: VCH Publishers, Inc. (Tachyons).

Ferrarese, Laura, \& Merritt, David. (2000). A Fundamental Relation between Supermassive Black Holes and their Host Galaxies. The Astrophysical Journal, 539, L9-L12. Retrieved from http://adsabs.harvard.edu/abs/2000ApJ539L9F

Folse, Henry J. (1995). Niels Bohr and the Construction of a New Philosophy. Studies in History and Philosophy of Science, 26(1), 107-116.

Freire, Olival Jr. (2006). Philosophy enters the optics laboratory: Bell's theorem and its first experimental tests (1965-1982). Studies in History and Philosophy of Science Part B: Studies In History and Philosophy of Modern Physics, 37(4), 577-616. http://dx.doi.org/10.1016/j.shpsb.2005.12.003

Fullat, O., \& Fontan, P. (1986). Historia de la Filosofia (1 ${ }^{\text {st }}$ Ed.). Spain: Editorial Vicens-Vives (Chapter 1)

Gabbatt, Adam. (2010). Stephen Hawking says universe not created by God. The Guardian. Guardian News and Media. Retrieved from http://www.guardian.co.uk/science/2010/sep/02/stephen-hawking-big-bang-creator

Genovesem, Marco. (2005). Research on hidden variable theories: A review of recent progresses. Physics Reports, 413(6), 319-396. http://0-dx.doi.org.lib.utep.edu/10.1016/j.physrep.2005.03.003

Glassman, Robert B. (1999). Hypothesized neural dynamics of working memory: Several chunks might be marked simultaneously by harmonic frequencies within an octave band of brain waves. Brain Research Bulletin, 50(2), 77-93. http://dx.doi.org/10.1016/S0361-9230(99)00090-8

Granatstein, Victor L. (1991). Encyclopedia of Physics (2 ${ }^{\text {nd }}$ Ed.). New York: VCH Publishers, Inc. (Light). 
Healey, Richard. (2003). Holism in philosophy of mind and philosophy of physics: Michael Esfeld, DordrechtStudies. Studies In History and Philosophy of Science Part B: Studies In History and Philosophy of Modern Physics, 34(2), 334-337. http://dx.doi.org/10.1016/S1355-2198(03)00011-X

Hilgevoord, J. (2005). Time in quantum mechanics: a story of confusion. History and Philosophy of Modern Physics. 36(1), 29-60. http://0-dx.doi.org.lib.utep.edu/10.1016/j.shpsb.2004.10.002

Honner, John. (1982). The Transcendental Phylosophy of Niels Bohr. Studies in History and Philosophy of Science, 13(1), 1-29. http://dx.doi.org/10.1016/0039-3681(82)90002-4

Jammer, Max. (2002). Concepts of mass in contemporary physics and philosophy. Studies In History and Philosophy of Science Part B: Studies In History and Philosophy of Modern Physics, 33(4), 730-735. http://0-dx.doi.org.lib.utep.edu/10.1016/S1355-2198(02)00036-9

Levine, Ira N. (1991). Quantum Chemistry (4 ${ }^{\text {th }}$ Ed.). New Yersey: Prentice-Hall, Inc.

Markosian, Ned. (2002). Time. The Stanford Encyclopedia of Philosophy. Retrieved from http://plato.stanford.edu/entries/time/\#3 $\quad$ (February 2012). http://dx.doi.org/10.1016/S1355-2198(02)00036-9

McGraw-Hill. (1994). Concise Encyclopedia of Science \& Technology (3rd Edition). United States of America: McGraw-Hill, Inc.

Morrison, \& Boyd. (1973). Organic Chemistry (3rd Edition). Boston: Allyn and Bacon, Inc.

Pankaj S. Joshi (2009). Do Naked Singularities Break the Rules of Physics? American Scientific. Retrieved from http://www.scientificamerican.com/article.cfm?id=naked-singularities

Planck, Max. (1944). The Nature of Matter. Speech at Florence, Italy.

Richardson, Alan. (2003). The geometry of knowledge: Lewis, Becker, Carnap and the formalization of philosophy in the 1920s. Studies in History and Philosophy of Science, 34, 165-182. http://dx.doi.org/10.1016/S0039-3681(02)00088-2

Rickles, D. (2010). The Ashgate Companion to Philosophy of Studies Physics. History and Philosophy of Science Part B: Studies In History and Philosophy of Modern Physics, 41(4), 362-365. http://0-dx.doi.org.lib.utep.edu/10.1016/j.shpsb.2010.04.001

Ruchvarger, H. (2011). Geometrical Correlation between Extra-dimensions and Universe Accelerated $\begin{array}{lllll}\text { Expansion. } & \text { Applied } & \text { Physics } & \text { Research. } & \text { 110-129. }\end{array}$ http://ccsenet.org/journal/index.php/apr/article/view/12785/9069

Rynasiewicz, Robert. (2004). Newton's Views on Space, Time, and Motion. Stanford Encyclopedia of Philosophy. Retrieved from http://plato.stanford.edu/entries/newton-stm/

Schummer, Joachim. (2003). The philosophy of chemistry. Endeavour. 27(1), 37-41. http://dx.doi.org/10.1016/S0160-9327(03)00004-8

Serway, Raymond A. (1986). Physics for Scientists \& Engineers $\left(2^{\text {nd }}\right.$ Ed.). New York: Saunders College Publishing (Chapter 29).

Wolf, Alan F. (1988). Parallel Universes. Simon \& Schuster.

Worsley, Andrew. (2012). Advances in Black Hole Physics and Dark Matter Modelling of the Galactic Halo. Applied Physics Research. 4(1), 128-137. http://dx.doi.org/10.5539/apr.v4n1p128

Yakushevich, Ludmila V. (2001). Is DNA a nonlinear dynamical system where solitary conformational waves are possible? Journal of Bioscience. 26(3). 305-313. http://dx.doi.org/10.1007/BF02703739

Zinkernagel, H. (2011). Some Trends in the Philosophy of Physics. Theoria, 71, 215-241. http://www.ehu.es/ojs/index.php/THEORIA/article/view/1853/1924 